\title{
Structure of $\mathrm{GeSe}_{4}-\mathrm{In}$ and $\mathrm{GeSe}_{5}$-In glasses
}

\author{
I Kaban $^{1^{*}}$, P Jóvári ${ }^{2}$, T Petkova ${ }^{3}$, Petkov ${ }^{4}$, A Stoilova ${ }^{4}$, W Hoyer ${ }^{1}$, B Beuneu ${ }^{5}$ \\ ${ }^{1}$ Institute of Physics, Chemnitz University of Technology, D-09107 Chemnitz, Germany \\ *Present address: IFW Dresden, Institute for Complex Materials, PB 270116, D-01171 \\ Dresden, Germany \\ ${ }^{2}$ Research Institute for Solid State Physics and Optics, H-1525 Budapest, POB 49, Hungary \\ ${ }^{3}$ Institute of Electrochemistry and Energy Systems, BAS, Acad. G. Bonchev B1.10, \\ 1113 Sofia, Bulgaria \\ ${ }^{4}$ Department of Physics, University of Chemical Technology and Metallurgy, 8 Kliment \\ Ohridsky Blvd, 1756 Sofia, Bulgaria \\ ${ }^{5}$ Laboratoire Léon Brillouin, CEA-Saclay 91191 Gif sur Yvette Cedex France \\ E-mail: ivan.kaban@physik.tu-chemnitz.de; i.kaban@ifw-dresden.de
}

\begin{abstract}
$\left(\mathrm{Ge}_{0.2} \mathrm{Se}_{0.8}\right)_{100-\mathrm{x}} \mathrm{In}_{\mathrm{x}}$ and $\left(\mathrm{Ge}_{0.17} \mathrm{Se}_{0.83}\right)_{100-\mathrm{x}} \mathrm{In}_{\mathrm{x}}(x=0,5,10,15$ at.\%) chalcogenide glasses have been studied with high-energy x-ray diffraction, neutron diffraction and extended x-ray absorption spectroscopy at $\mathrm{Ge}$, Se and In $K$-edges. The experimental data were modelled simultaneously with the reverse Monte-Carlo simulation method. $\mathrm{GeSe}_{4 / 2}$ tetrahedra are shown to be the main structural units in the binary and ternary glasses investigated. Indium bonds to the excess Se atoms in the ternary Ge-Se-In glasses. While majority of In atoms have three Se neighbours, some In atoms may be tetrahedrally coordinated by Se.
\end{abstract}

PACS numbers: 61.43.Dq, 61.05.cp, 61.05.fm, 61.05.cj

(Figures in this article are in colour only in the electronic version)

\section{Introduction}

Chalcogenide glasses have found use in various applications such as information storage devices, chemical sensor systems, optical transmitters and amplifiers [1-5]. The physical properties of amorphous chalcogenides can be easily varied by addition of some metals or semimetals that enables extending the application field. For example, introduction of $\mathrm{Bi}$ or $\mathrm{Pb}$ into $\mathrm{Ge}-\mathrm{Se}$ glass induces the change of electrical conduction type from $p$ to $n$, the decrease of electrical resistivity, and the change 
of optical properties [6,7]. On the other hand, amorphous Ge-Se-Ag alloys are characterized by a very high electrical conductivity with essential ionic component [8]. Also, the charge carrier concentration increases remarkably upon addition of $\mathrm{Cu}$ into $\mathrm{Ge}-\mathrm{Se}$ glass [9]. This makes possible using chalcogenide glasses as membranes in ion selective electrodes for detection of metals in aqueous solutions [10]. Photoconductivity, thermal diffusivity and the optical band gap of chalcogenide glasses can be considerably changed by alloying of a third element to Ge-Se [11-14]. For, example, addition of $\mathrm{B}, \mathrm{Ga}$ or $\mathrm{Tl}$ causes an increase of the optical band gap $E_{\mathrm{g}}$ [11]. The optical band gap decreases and photoconductivity increases if $\mathrm{In}$ or $\mathrm{Cd}$ is added to Ge-Se [12-15].

Based on analysis of the composition dependences for electrical conductivity, photocurrent, optical absorbance spectra and X-ray diffraction patterns of glassy $\left(\mathrm{Ge}_{0.28} \mathrm{Se}_{0.72}\right)_{95} \mathrm{In}_{5}$ and $\left(\mathrm{Ge}_{0.28} \mathrm{Se}_{0.72}\right)_{95} \mathrm{Cd}_{5} \mathrm{Bakr}$ [13] suggested that the main glass structure of $\mathrm{Ge}-\mathrm{Se}$ is interrupted by bonding of In or Cd with the dangling Se atoms. Petkov and Petkova [16] studied far-infrared and Raman spectra of Ge-Se-Tl thin films and supposed that the films are basically composed of $\mathrm{GeSe}_{4 / 2}$ tetrahedra, and $\mathrm{Tl}$ atoms link Se chains without any influence on the Ge-Se bonding. It has been suggested [14] that the changes in the glass structure upon addition of In to Ge-Se are associated with Se chains shortening and an increase in defect concentration. This results in formation of new localized states in the band structure and a decrease of the optical band gap.

Ge-Se-In alloys are good glass formers over a wide compositional region as reported by Boncheva et al [17]. Along the quasibinary $\mathrm{GeSe}_{4}-\mathrm{In}$ and $\mathrm{GeSe}_{5}$-In tie-lines, amorphisation is possible up to 20 mol.\% In. Ge-Se-In glasses do not show any phase separation neither after preparation nor after annealing at temperatures under the glass transition temperature for a given composition. Heating above the glass transition temperature but below crystallization temperature facilitates the appearance of the crystalline phases $\mathrm{In}_{2} \mathrm{Se}_{3}$ and $\mathrm{In}_{6} \mathrm{Se}_{7}$ as reported in [18].

The atomic structure of Ge-Se-In chalcogenide glasses is not as well studied as their physical properties. Ledru et al [19] found that the extended x-ray absorption fine structure (EXAFS) spectra at indium $K$ absorption edge of Ge-Se-In glasses are virtually the same for different compositions. Fitting the first coordination shell, they established that each In atom has on average three Se nearest neighbours at the distance of about 2.6 ̊. However, Kosek et al [20] suggested that indium atoms can also be tetrahedrally coordinated in Ge-Se-In glasses.

In the present work we perform an experimental and modelling study of the structure of ternary Ge-Se-In glasses based on the binary $\mathrm{GeSe}_{4}$ and $\mathrm{GeSe}_{5}$ compositions. X-ray diffraction (XRD), neutron diffraction (ND), and EXAFS experimental techniques and reverse Monte Carlo (RMC) simulation method have been applied. Simultaneous modelling of several experimental datasets for each composition enabled obtaining partial pair distribution functions and extracting information on the local atomic distribution in glasses. 


\section{Experimental details}

Chalcogenide glasses with the nominal compositions $\left(\mathrm{Ge}_{0.2} \mathrm{Se}_{0.8}\right)_{100-\mathrm{x}} \mathrm{I} \mathrm{n}_{\mathrm{x}}$ and $\left(\mathrm{Ge}_{0.17} \mathrm{Se}_{0.83}\right)_{100-\mathrm{x}} \mathrm{In}_{\mathrm{x}}(x=0$, 5, 10 and 15 at.\%) were prepared from elemental Ge, Se and In of $99.99 \%$ purity. For convenience, we will further denote the alloys as $\mathrm{GeSe}_{4}-\mathrm{In}_{\mathrm{x}}$ and $\mathrm{GeSe}_{5}-\mathrm{In}_{\mathrm{x}}$ respectively. The proper quantities of alloy constituents were sealed in quartz ampoules under residual pressure of $10^{-3} \mathrm{~Pa}$. The ampoules were heated in a furnace with a rate of $2 \mathrm{~K} / \mathrm{min}$ up to $1273 \mathrm{~K}$ applying vibration stirring. Then the samples were rapidly quenched in a mixture of water and ice.

The XRD experiments were carried out at the BW5 experimental station [21] at HASYLAB, DESY (Hamburg, Germany). The samples were powdered and filled into thin walled $(0.02 \mathrm{~mm})$ quartz capillaries of $2.0 \mathrm{~mm}$ inner diameter. The energy of the incident beam was $100 \mathrm{keV}$. The size of the incident beam was $1 \times 4 \mathrm{~mm}^{2}$. The scattered intensity was recorded by a Ge solid-state detector. The raw data were corrected for background, absorption, polarization, detector dead-time and variations in detector solid angle [22].

The EXAFS measurements were carried out on all samples at Ge, Se and In K-edges at the beam line X [21] of HASYLAB in transmission mode. The samples were finely ground, mixed with cellulose and pressed into tablets. The sample quantities in the tablets were adjusted to the alloy compositions and to the selected edges. The transmission of the samples was about 1/e. The EXAFS spectra were obtained with the step $0.5 \mathrm{eV}$ in the vicinity of the absorption edge. The measuring time was $k$-weighted during the collection of the signal.

The ND measurement on $\mathrm{GeSe}_{5}-\mathrm{In}_{15}$ glass was carried out at the $7 \mathrm{C} 2$ diffractometer at the Léon Brillouin Laboratory, CEA-Saclay, France. The sample was packed into thin walled $(0.1 \mathrm{~mm})$ vanadium container with $7 \mathrm{~mm}$ diameter. The raw data were corrected for detector efficiency, empty instrument background, scattering from the sample holder, multiple and incoherent scattering, and absorption.

\section{Experimental results}

The experimental X-ray and neutron scattering intensities were converted into the total structure factors using Faber-Ziman $[23,24]$ formalism. The total structure factor $S(Q)$ of an alloy is related to the partial structure factors $S_{\mathrm{ij}}(Q)$ by the relationship:

$$
S(Q)=\sum_{i \leq j} w_{i j}^{X, N}(Q) S_{i j}(Q)
$$

Here, $Q$ is the magnitude of the scattering vector $Q=4 \pi \sin \theta / \lambda$ ( $\theta$ is half the scattering angle and $\lambda$ is the wavelength used in the scattering experiment). In the Faber-Ziman formalism, the weights $w_{i j}$ are expressed as 


$$
w_{i j}^{X}(Q)=\left(2-\delta_{i j}\right) c_{i} c_{j} \frac{f_{i}(Q) f_{j}(Q)}{\langle f(Q)\rangle^{2}}, \quad w_{i j}^{N}=\left(2-\delta_{i j}\right) c_{i} c_{j} \frac{b_{i} b_{j}}{\langle b\rangle^{2}}
$$

where the upper index $X$ stands for XRD and index $N$ stands for ND; $\delta_{i j}$ is the delta symbol of Kronecker; $c_{i}$ is the mole fraction of the $i$-th constituent; $f_{i}(Q)$ is the atomic form factor and $b_{i}$ is the coherent neutron scattering length. The Faber-Ziman partial structure factors $S_{i j}(Q)$ are linked to the partial pair correlation functions $g_{i j}(r)$ by the expression:

$$
g_{i j}(r)-1=\frac{1}{2 \pi^{2} \rho_{0} r} \int_{0}^{\infty} Q\left(S_{i j}(Q)-1\right) \sin (Q r) d Q
$$

or

$$
S_{i j}(Q)=1+\frac{4 \pi \rho_{0}}{Q} \int r \sin Q r\left(g_{i j}(r)-1\right) d r
$$

where $\rho_{0}$ is the average number density of the alloy.

The experimental energy dependences of the X-ray absorption coefficient $\mu(E)$ are converted into the EXAFS functions $\chi(E)$ with the expression:

$$
\chi(E)=\frac{\mu(E)-\mu_{0}(E)}{\Delta \mu_{0}(E)} .
$$

Here $\mu_{0}(E)$ is a smooth background function representing the absorption of an isolated atom, and $\Delta \mu_{0}(E)$ is the measured jump in the absorption $\mu(E)$ at the absorption edge $E_{0}$. As the EXAFS is best understood in terms of the wave behaviour of the photo-electron created in the absorption process, it is common to present the EXAFS spectra as a function of the wave number $k$ of the photoelectron ( $k=\sqrt{2 m_{e}\left(E-E_{0}\right) / \hbar} ; m_{\mathrm{e}}$ is the electron mass, and $\hbar$ is the reduced Planck constant). The details on EXAFS can be found for example at the site for the X-ray absorption spectroscopy [25] or in the Ref. [26]. In the present work, the experimental EXAFS data were treated with the VIPER program [27].

The experimental total structure factors obtained with XRD and the corresponding pair distribution functions for the $\mathrm{GeSe}_{4}$-In and $\mathrm{GeSe}_{5}$-In glasses are plotted in Figs. 1 and 2. Figure 3 shows as an example the whole sets of the experimental data - XRD, ND and EXAFS - for the ternary $\mathrm{GeSe}_{5}-\mathrm{In}_{15}$ glass. To emphasize the EXAFS oscillations, which decay quickly with $k$, the $\chi(k)$ spectra are multiplied by the $k^{3}$.

The effect of In on the structure of ternary Ge-Se-In glasses is well reflected on the XRD total pair distribution functions plotted in Figs. $1 \mathrm{~b}$ and $2 \mathrm{~b}$. Remarkable is the decrease of the first maximum of the $g(r)$ functions (peak at $r=2.36 \AA$, Figs. $1 \mathrm{~b}$ and $2 \mathrm{~b}$ ) and appearance of a shoulder on its right 
side which develops into a new peak at $2.6 \AA$ for 15 at.\% In. The first peak on the $g(r) \mathrm{s}$ reflects $\mathrm{Ge}-\mathrm{Se}$ and Se-Se bonding. Indeed, Ge-Se nearest neighbour distance of $2.36 \pm 0.02 \AA$ and Se-Se distance of $2.32 \pm 0.02 \AA$ were determined in the ND study of Ge-Se glasses with isotopic substitution in [28]. The shoulder/peak at $2.6 \AA$ can be related to the In-Se pairs as this value $(2.6 \AA)$ agrees with the sum of covalent radii for In and Se [29] and with the In-Se distance in ternary Ge-Se-In glasses found by Ledru et al with EXAFS [19]. As it has been shown in the studies of binary Ge-Se glasses (e.g. Ref. [28] and citations therein), Se atoms are in excess in the alloys with Se concentration $>2 / 3$. The area under the first peak on the total pair distribution functions for the ternary compositions (Figs. 1b and $2 b)$ reduces simultaneously with formation of the In-Se bonds. It is reasonable to suppose that $\mathrm{GeSe}_{4 / 2}$ units remain intact in the ternary glasses as long as Se atoms are in excess, and the formation of In-Se bonds is at the expense of Se-Se and not Ge-Se bonds. All these suppositions can be verified if the partial pair distribution functions are known. For this purpose, the reverse Monte-Carlo modelling technique has been used.

\section{Reverse Monte-Carlo modelling of Ge-Se-In glasses}

\subsection{RMC simulation details}

In the present work, atomic structures of Ge-Se-In glasses have been modelled with the new RMCPP code [30]. The simulation boxes contained 18000 atoms. The number densities $\rho$ used in the simulations (Table 1) were calculated by compilation of the experimental mass densities reported in the literature [1,31] and own measurements (unpublished). The minimum interatomic distances (cut offs) applied in the final simulation runs (used to produce configurations for further analysis) are listed in Table 2. Initial configurations were obtained via the following steps: 1) random atomic coordinates were generated; 2) atoms were moved to satisfy cut off constraints. Then several simulation runs were carried out to test the validity of the cut offs (e.g. the necessity of Ge-In bonds was checked by lowering the Ge-In cut off to $2.4 \AA$ ). Finally it was concluded that by assuming Ge-Se, Se-Se and SeIn bonds all measurements could be fitted properly. In the next step coordination constraints were applied (see below).

The backscattering amplitudes and phases needed to obtain the model EXAFS curves from the pair distribution functions were calculated by the FEFF8.4 program [32]. In line with the pair correlation formalism of RMC only two-atom calculations were carried out (one absorber and one backscatter). This approximation proved to be adequate far above the absorption edge $\left(k \geq 4.5 \AA^{-1}\right.$ or about $80-85 \mathrm{eV}$ above the edge) where the contribution of multiple scattering processes to EXAFS spectra becomes negligible (see e.g. [33]). The absorber-backscatterer distance was changed in $0.1 \AA$ steps. This binning was sufficient to reproduce EXAFS datasets up to the highest $k$ values measured. The minimum distance used in these calculations coincided with the corresponding cut offs, while the maximum separation was equal to the first minimum of the pair correlation functions. For more details 
of fitting simultaneously diffraction and EXAFS datasets with the reverse Monte Carlo method we refer to some recent publications [30,33,34].

Fits obtained by the simultaneous modelling of XRD, ND as well as Ge-, Se- and In K-edge EXAFS measurements for the glass $\mathrm{GeSe}_{5}-\mathrm{In}_{15}$ are compared with the experimental data in Fig. 3. For the other compositions XRD, Ge-, Se- and In K-edge EXAFS data were fitted with a quality similar to that of Fig. 3 (not shown). The partial pair distribution functions $g_{\mathrm{ij}}(r)$ corresponding to the model configurations for the $\mathrm{GeSe}_{4}$-In and $\mathrm{GeSe}_{5}$-In glasses are presented in Figs. 4 and 5. The corresponding mean nearest neighbour distances $r_{\mathrm{ij}}$ and coordination numbers $N_{\mathrm{ij}}$ are presented in Tables 3 and 4 . It should be noted that only neighbours within the first coordination shell are considered. The uncertainty of $r_{\mathrm{ij}}$ is usually around $\pm 0.02 \AA$ but it can be significantly higher $(0.05-$ $0.1 \AA$ ) for atomic pairs with low contribution to the total pair distribution function. The error of the total coordination number for alloy constituents $N_{i X}$ is around 5-10\%, while that of partial coordination numbers $N_{\mathrm{ij}}$ can again be higher (up to $15-20 \%$ for unconstrained coordination numbers).

\subsection{Binary $\mathrm{GeSe}_{4}$ and $\mathrm{GeSe}_{5}$ glasses}

The structure of Ge-Se binary glasses has been extensively investigated so far (a detailed list of publications can be found, for example, in the paper of Salmon [28]). All studies point to the existence of $\mathrm{GeSe}_{4 / 2}$ tetrahedra as basic structural units in the binary Ge-Se glasses. The number of possible homonuclear bonds $\mathrm{Ge}-\mathrm{Ge}$ and $\mathrm{Se}-\mathrm{Se}$ is a minimum in the stoichiometric $\mathrm{GeSe}_{2}$ glass ( $N_{\mathrm{GeGe}}=0.25 \pm 0.05$ and $N_{\mathrm{SeSe}}=0.20 \pm 0.05$ were found in Ref. [28]) and increases in the compositions either side of $\mathrm{GeSe}_{2}$.

In the present work, the following values have been obtained with the reverse Monte-Carlo modelling. In the $\mathrm{GeSe}_{4}$ glass, each Ge atom is coordinated by about four Se atoms, while Se atoms have both $\mathrm{Ge}$ and Se neighbours $\left(N_{\mathrm{SeGe}}=1, N_{\mathrm{SeSe}} \approx 1.1\right.$, Table 3$)$. In the $\mathrm{GeSe}_{5}$ glass, each Ge atom is coordinated by about four Se atoms, while Se atoms also have both Ge and Se neighbours $\left(N_{\mathrm{SeGe}}=0.8\right.$, $N_{\text {SeSe }} \approx 1.3$, Table 4).

\subsection{Ternary $\mathrm{GeSe}_{4}$-In and $\mathrm{GeSe}_{5}$-In glasses}

As the ternary Ge-Se-In glasses studied in the present work are based on the binary $\mathrm{GeSe}_{4}$ and $\mathrm{GeSe}_{5}$ compositions, existence of Ge-Ge homonuclear bonds there can be safely excluded. Also, it is very improbable that In can bond to Ge when all Ge atoms are already coordinated by Se atoms. Finally, due to the small concentrations of In, formation of In-In homonuclear bonds is also unexpected. As it has been mentioned, Ledru et al [19] found in their EXAFS study that In atoms are bonded to Se in ternary Ge-Se-In glasses. Thus, only Ge-Se, Se-Se and In-Se bonds were allowed in the RMC simulations. Assuming that the basic behaviour of Ge and Se in the Ge-Se-In glasses is the same as in 
the binary Ge-Se glasses, the following coordination constraints were used: $N_{\mathrm{GeSe}}=4 ; N_{\mathrm{SeSe}}+N_{\mathrm{SeGe}}=$ $0,1,2$ for each Se (that is, the number of Se and Ge atoms around a Se atom cannot increase upon adding In). $N_{\text {Inse }}$ cannot be 0 or 1 for each In.

With these constraints, the pair distribution functions presented in Figs. 4 and 5 and the structural parameters listed in Tables 3 and 4 have been obtained. The shape of the first peaks on the model pair distribution functions suggests that the peak positions are determined by the experimental data and not by the constraints. Indeed, the position of Ge-Se and Se-Se peaks is much higher than the cut offs applied though in some cases small spikes can be found on the low- $r$ side of the peaks. The position of these spikes was sensitive to the choice of the cut offs. In most cases it was possible to merge them into the main peak. However, sometimes it was not possible to eliminate them simultaneously from $g_{\text {Gese }}(r)$ and $g_{\text {Sese }}(r)$. As the uncertainty caused by the spikes is rather small and it does not influence the conclusions of the paper this highly methodological question was not addressed in detail.

Due to their similar size and scattering properties it is not possible to separate $\mathrm{Ge}$ and $\mathrm{Se}$ merely on the basis of experimental information (except neutron diffraction with isotopic substitution which is, unfortunately, prohibitively expensive for larger series of samples). Thus, the similarity of $g_{\mathrm{GeGe}}(r)$ and $g_{\mathrm{SeSe}}(r)$ beyond the first coordination sphere - where coordination constraints do not apply - may be regarded as an artefact. However, the second peak of a pair correlation function in a covalent network is mostly due to the second neighbour distances. In case of $g_{\text {GeGe }}(r)$ and $g_{\text {Sese }}(r)$ these distances are determined by the corresponding nearest neighbour distances $\left(r_{\mathrm{GeSe}}\right.$ and $\left.r_{\mathrm{SeSe}}\right)$ and bond angles (Ge-Se-Ge for $g_{\mathrm{GeGe}}(r)$ and Se-Se-Se and Se-Ge-Se for $\left.g_{\mathrm{SeSe}}(r)\right)$ which all have quite similar values (2.35-2.37 $\AA$ for the distances and $100-109^{\circ}$ for the angles). Therefore, we believe that the coincidence of the second peak positions on $g_{\mathrm{GeGe}}(r)$ and $g_{\mathrm{SeSe}}(r)$ is not necessarily an artefact, especially if we take into account the different amplitudes of the peaks.

Usually, around $95 \%$ of atoms satisfied coordination constraints. Thus, $N_{\mathrm{Ge}}=N_{\mathrm{GeSe}}=4$ was forced for all ternary compositions studied. This yields, $N_{\mathrm{SeGe}}=1$ for the $\mathrm{GeSe}_{4}-\mathrm{In}_{\mathrm{x}}$ series and $N_{\mathrm{SeGe}}=$ 0.8 for the $\mathrm{GeSe}_{5}-\mathrm{In}_{\mathrm{x}}$ series. The following coordination numbers, which were unconstrained, are found due to the RMC modelling both in the $\mathrm{GeSe}_{4}-\mathrm{In}_{\mathrm{x}}$ and $\mathrm{GeSe}_{5}-\mathrm{In}_{\mathrm{x}}$ glasses: $N_{\mathrm{SeSe}}=0.9-1, N_{\mathrm{In}}=$ $N_{\text {InSe }}=3.3-3.5, N_{\text {SeIn }}=0.2,0.5,0.7$ for $5,10,15 \%$ In.

According to our model, Ge and Se form $\mathrm{GeSe}_{4 / 2}$ tetrahedra, while In atoms bind to the Se atoms which are in excess. As the mean Se-Se coordination number decreases from 1.1-1.2 in the binary $\mathrm{GeSe}_{4}$ and $\mathrm{GeSe}_{5}$ glasses down to 0.9-1, it can be suggested that indium breaks some $\mathrm{Se}-\mathrm{Se}$ pairs and intrudes between the Se atoms and bonds them. The mean coordination number $N_{\text {InSe }}$ obtained in the present study (3.3 - 3.5) is somewhat above the value found in the EXAFS study of Ledru et al [19] $\left(N_{\mathrm{InSe}}=3\right)$. Actually, this difference (0.3-0.5) is comparable with uncertainty of the coordination number for a minority component. However, the uncertainty decreases with increasing In concentration. Therefore, it is more probable that, while majority of In atoms is three-fold coordinated 
by Se, some In atoms get four Se neighbours. As it has been mentioned, tetrahedral coordination of In in Ge-Se-In glasses has been supposed by Kosek et al [20]. Besides, it should be taken into account that indium is four- and fivefold coordinated in the crystalline $\gamma$ - $\operatorname{In}_{2} \mathrm{Se}_{3}$ [35].

Figure 6 shows the Se-Ge-Se and Se-In-Se bond angle distributions for the binary $\mathrm{GeSe}_{4}$ and ternary $\left(\mathrm{Ge}_{0.2} \mathrm{Se}_{0.8}\right)_{100-\mathrm{x}} \mathrm{In}_{\mathrm{x}}$ glasses extracted from the final model atomic configurations. The bond angle distributions are practically independent of the In content. As it can be expected, the peak of the Se-Ge-Se bond angle distribution $\left(\sim 105^{\circ}\right)$ is close to the tetrahedral angle $\left(109.47^{\circ}\right)$. The main peak of the Se-In-Se bond angle distribution is around $95^{\circ}$ with a tail towards $180^{\circ}$. A similar picture (not shown) is observed in the $\left(\mathrm{Ge}_{0.17} \mathrm{Se}_{0.83}\right)_{100-\mathrm{x}} \mathrm{In}_{\mathrm{x}}$ glasses. These observations confirm our supposition derived from the analysis of coordination numbers that In atoms do not affect strongly the $\mathrm{GeSe}_{4 / 2}$ tetrahedra, but bind Se atoms which are in excess.

\section{Conclusions}

Atomic structure of Ge-Se and Ge-Se-In chalcogenide glasses with the constant ratio $\mathrm{Ge}: \mathrm{Se}=4$ or 5 and containing up to 15 atomic percent of In has been studied experimentally and modelled using the reverse Monte-Carlo simulation technique. According to our model, Ge is bonded to four Se nearest neighbours forming $\mathrm{GeSe}_{4 / 2}$ tetrahedral structural units in all binary and ternary glasses investigated. Consequently, each Se atom has one Ge nearest neighbour in the $\mathrm{GeSe}_{4}-\mathrm{In}_{\mathrm{x}}$ alloys, while the average Se-Ge coordination is 0.8 in the $\mathrm{GeSe}_{5}-\mathrm{In}_{\mathrm{x}}$ compositions. The excess Se atoms make homonuclear bonds in the binary glasses $\mathrm{GeSe}_{4}\left(N_{\mathrm{SeSe}} \approx 1.1\right)$ and $\mathrm{GeSe}_{5}\left(N_{\mathrm{SeSe}} \approx 1.3\right)$. In the ternary Ge-Se-In glasses, indium bonds to selenium, probably by breaking some Se-Se pairs. The mean coordination number of Se increases from 2 to about 2.5 upon increasing In concentration in Ge-Se-In glasses. The average coordination number of In is about 3.3-3.5 suggesting that while majority of In atoms have three neighbours some In atoms are tetrahedrally coordinated by Se.

\section{Acknowledgments}

This study has been supported by Bulgarian Ministry of Education and Science and German Academic Exchange Service (DAAD). P. Jóvári was supported by the Bolyai Research Fellowship of the Hungarian Academy of Sciences. I. Kaban thanks DESY for the support of XRD and EXAFS measurements at HASYLAB (Hamburg, Germany). The experiment at LLB was supported by the European Commission through the Access Activities of the Integrated Infrastructure Initiative for Neutron Scattering and Muon Spectroscopy (NMI3), supported by the European Commission under the 7th Framework Programme through the Key Action: Strengthening the European Research Area, Research Infrastructures, Contract NMI3/FP7 no: 226507. 


\section{References}

[1] Borisova Z U 1981 Glassy Semiconductors, New York, Plenum.

[2] Kokorina V F 1996 Glasses for Infrared Optics, Boca Raton, CRC Press.

[3] Yamada N and Matsunaga T 2000 J. Appl. Phys. 887020.

[4] Mourzina Y, Yoshinobu T, Schubert J, Lüth H, Iwasaki H, Schöning M J 2001 Sensors and Actuators B80 136.

[5] Zakery A and Elliott S R 2003 J. Non-Cryst. Solids 3301.

[6] Tohge N, Minami T, Yamamoto Y and Tanaka M 1980 J. Appl. Phys. 511048.

[7] Ganesan R 2004 Thermal and optical properties of Ge-Se glass matrix doped with Te, Bi and Pb, $\mathrm{PhD}$ thesis, Indian Institute of Science, Bangalore, India; http://hdl.handle.net/2005/337

[8] Angel C A 1992 Annu. Rev. Phys. Chem. 43693.

[9] Thakur A, Sainty G S S, Goyal N and Thripathi S K 2007 J. Non-Cryst. Solids 3531326.

[10] Conde Garrido J M, Andrea Ureña M, Arcondo B 2010 J. Alloys Comp. 495356.

[11] Petkov P 2009 in Nanostructured Materials for Advanced Technological Applications, eds. Reithmaier J P, Petkov P, Kulisch W and Popov C, Dordrecht, Springer Netherlands, p.315. (doi: 10.1007/978-1-4020-9916-8_34)

[12] Batrakov Yu, Zaitsev Yu M, Gutenev M S, Karpova E A, Makarov L L and Khramtsov K G 1985 Theoret. Experim. Chem. 20646.

[13] Bakr N A 2003 J. Mater. Process. Techn. 132138.

[14] Petkov P, Stoilova A, Nedeva Y, Petkov E 2010 Surf Interf Analysis 421235.

[15] Mathew G and Philip J 1999 J. Phys. 115283.

[16] Petkov P and Petkova T 2000 Semicond. Sci. Technol. 15331.

[17] Boncheva-Mladenova Z, Mitkova M, Ivanova Z 1973 Compt. Rend. Acad. Bulg. Sci. 26671.

[18] El-Sayed M. Farag (2004) J. Mater. Sci. Mater.Electron 1519.

[19] Ledru J, Saiter J M, Saffarini G and Benazeth S 1998 J. Non-Cryst. Solids 232-234 634.

[20] Kosek F, Cimpl Z, Mikhailov M, Karpova EA 1986 J. Non-Cryst. Solids 86265.

[21] http://www.hasylab.de

[22] Poulsen H F, Neumann H-B, Schneider J R, Neuefeind J and Zeidler M D 1995 J. Non-Cryst.

Solids 18863.

[23] Faber T E and Ziman J M 1965 Phil. Mag. 11, 153.

[24] Waseda Y 1980 The Structure of Non-Crystalline Materials (McGraw-Hill, New York).

[25] http://www.xafs.org; Newville M 2004 Fundamentals of XAFS, Consortium for Advanced Radiation Sources, University of Chicago, Chicago, IL.

$\mathrm{http}: / /$ www.xafs.org/Tutorials?action=AttachFile \&do=get\&target=Newville_xas_fundamentals.pdf [26] Rehr J J and Albers R C 2000 Rev.Mod. Phys. 72, 621.

[27] Klementev K V (2001) J. Phys. D: Appl. Phys. 34, 209.

[28] Salmon P S 2007 J. Non-Cryst. Solids 3532959. 
[29] http://www.webelements.com

[30] Gereben O, Jóvári P, Temleitner L and Pusztai L 2007 J. Optoelectron. Adv. Mater. 93021.

[31] Saffarini G 1999 Phys. Stat. Sol. 213261.

[32] Ankudinov A L, Ravel B, Rehr J J and Conradson S D 1998 Phys. Rev. B58, 7565.

[33] Kaban I, Jóvári P, Hoyer W and Welter E 2007 J. Non. Cryst. Solids 3532474.

[34] Jóvári P,, Yannopoulos S N, Kaban I, Kalamponias A, Lishchynskyy I, Beuneu B, Kostadinova O, Welter E and Schöps A 2008 J. Chem. Phys. 129214502.

[35] Lutz H D, Fischer M, Baldus H-P and Blachnik R (1988) J. Less Comm. Met. 14383. 
Table 1. Number density of $\left(\mathrm{Ge}_{0.2} \mathrm{Se}_{0.8}\right)_{100-\mathrm{x}} \mathrm{In}_{\mathrm{x}}$ and $\left(\mathrm{Ge}_{0.17} \mathrm{Se}_{0.83}\right)_{100-\mathrm{x}} \mathrm{I} \mathrm{n}_{\mathrm{x}}$ glasses used in the RMC simulations

\begin{tabular}{|l|l|l|l|l|}
\hline \multirow{2}{*}{ System } & \multicolumn{4}{|c|}{ In concentration (at.\%) } \\
\cline { 2 - 5 } & 0 & 5 & 10 & 15 \\
\hline GeSe $_{4}$-In & $32.8 \mathrm{~nm}^{-3}$ & $33.5 \mathrm{~nm}^{-3}$ & $34.1 \mathrm{~nm}^{-3}$ & $34.7 \mathrm{~nm}^{-3}$ \\
\hline $\mathrm{GeSe}_{5}$-In & $33.8 \mathrm{~nm}^{-3}$ & $33.7 \mathrm{~nm}^{-3}$ & $33.7 \mathrm{~nm}^{-3}$ & $33.4 \mathrm{~nm}^{-3}$ \\
\hline
\end{tabular}

Table 2. Minimum interatomic distances (cut offs) applied in the RMC simulation of Ge-Se-In glasses.

\begin{tabular}{|l|l|l|l|l|l|l|}
\hline Pair & Ge-Ge & Ge-Se & Ge-In & Se-Se & Se-In & In-In \\
\hline Cut off $(\AA)$ & 3.0 & 2.1 & 3.1 & 2.1 & 2.4 & 3.3 \\
\hline
\end{tabular}

Table 3. Mean interatomic distances $r_{\mathrm{ij}}$ and coordination numbers $N_{\mathrm{ij}}$ for $\left(\mathrm{Ge}_{0.2} \mathrm{Se}_{0.8}\right)_{100-\mathrm{x}} \mathrm{In}_{\mathrm{x}}$ glasses obtained with RMC modelling. Ge-Ge, Ge-In and In-In pairs were not allowed in the models.

\begin{tabular}{|l|l|l|l|l|l|l|l|l|}
\hline $\mathrm{X}_{\mathrm{In}}$ & \multicolumn{3}{|c|}{0} & \multicolumn{2}{c|}{5} & \multicolumn{2}{c|}{10} & \multicolumn{2}{c|}{15} \\
\hline Pairs & $r_{\mathrm{ij}}(\AA)$ & $N_{\mathrm{ij}}$ & $r_{\mathrm{ij}}(\AA)$ & $N_{\mathrm{ij}}$ & $r_{\mathrm{ij}}(\AA)$ & $N_{\mathrm{ij}}$ & $r_{\mathrm{ij}}(\AA)$ & $N_{\mathrm{ij}}$ \\
\hline Ge-Se & 2.37 & 3.87 & 2.36 & 3.92 & 2.36 & 3.99 & 2.36 & 3.89 \\
\hline Se-Ge & 2.37 & 0.97 & 2.36 & 0.98 & 2.36 & 1.0 & 2.36 & 0.97 \\
\hline Se-Se & 2.35 & 1.12 & 2.34 & 1.01 & 2.32 & 0.92 & 2.36 & 0.95 \\
\hline Se-In & - & - & 2.56 & 0.22 & 2.57 & 0.46 & 2.58 & 0.73 \\
\hline In-Se & - & - & 2.56 & 3.39 & 2.57 & 3.29 & 2.58 & 3.33 \\
\hline Ge-X & - & 3.87 & - & 3.92 & - & 3.99 & - & 3.89 \\
\hline Se-X & - & 2.09 & - & 2.23 & - & 2.38 & - & 2.65 \\
\hline In-X & - & - & - & 3.39 & - & 3.29 & - & 3.33 \\
\hline
\end{tabular}

Table 4. Mean interatomic distances $r_{\mathrm{ij}}$ and coordination numbers $N_{\mathrm{ij}}$ for $\left(\mathrm{Ge}_{0.17} \mathrm{Se}_{0.83}\right)_{100-\mathrm{x}} \mathrm{In}_{\mathrm{x}}$ glasses obtained with RMC. Ge-Ge, Ge-In and In-In pairs were not allowed in the models.

\begin{tabular}{|l|l|l|l|l|l|l|l|l|}
\hline $\mathrm{X}_{\mathrm{In}}$ & \multicolumn{3}{|c|}{0} & \multicolumn{3}{c|}{5} & \multicolumn{3}{c|}{10} & \multicolumn{2}{c|}{15} \\
\hline Pairs & $r_{\mathrm{ij}}(\AA)$ & $N_{\mathrm{ij}}$ & $r_{\mathrm{ij}}(\AA)$ & $N_{\mathrm{ij}}$ & $r_{\mathrm{ij}}(\AA)$ & $N_{\mathrm{ij}}$ & $r_{\mathrm{ij}}(\AA)$ & $N_{\mathrm{ij}}$ \\
\hline Ge-Se & 2.37 & 3.97 & 2.37 & 3.93 & 2.37 & 3.99 & 2.37 & 4.01 \\
\hline Se-Ge & 2.37 & 0.79 & 2.37 & 0.79 & 2.37 & 0.80 & 2.37 & 0.80 \\
\hline Se-Se & 2.34 & 1.32 & 2.33 & 1.02 & 2.34 & 1.04 & 2.33 & 0.92 \\
\hline Se-In & - & - & 2.57 & 0.21 & 2.57 & 0.47 & 2.58 & 0.74 \\
\hline In-Se & - & - & 2.57 & 3.40 & 2.57 & 3.55 & 2.58 & 3.52 \\
\hline Ge-X & - & 3.97 & - & 3.93 & - & 3.94 & - & 4.08 \\
\hline Se-X & - & 2.11 & - & 2.23 & - & 2.31 & - & 2.46 \\
\hline In-X & - & - & - & 3.40 & - & 3.55 & - & 3.52 \\
\hline
\end{tabular}



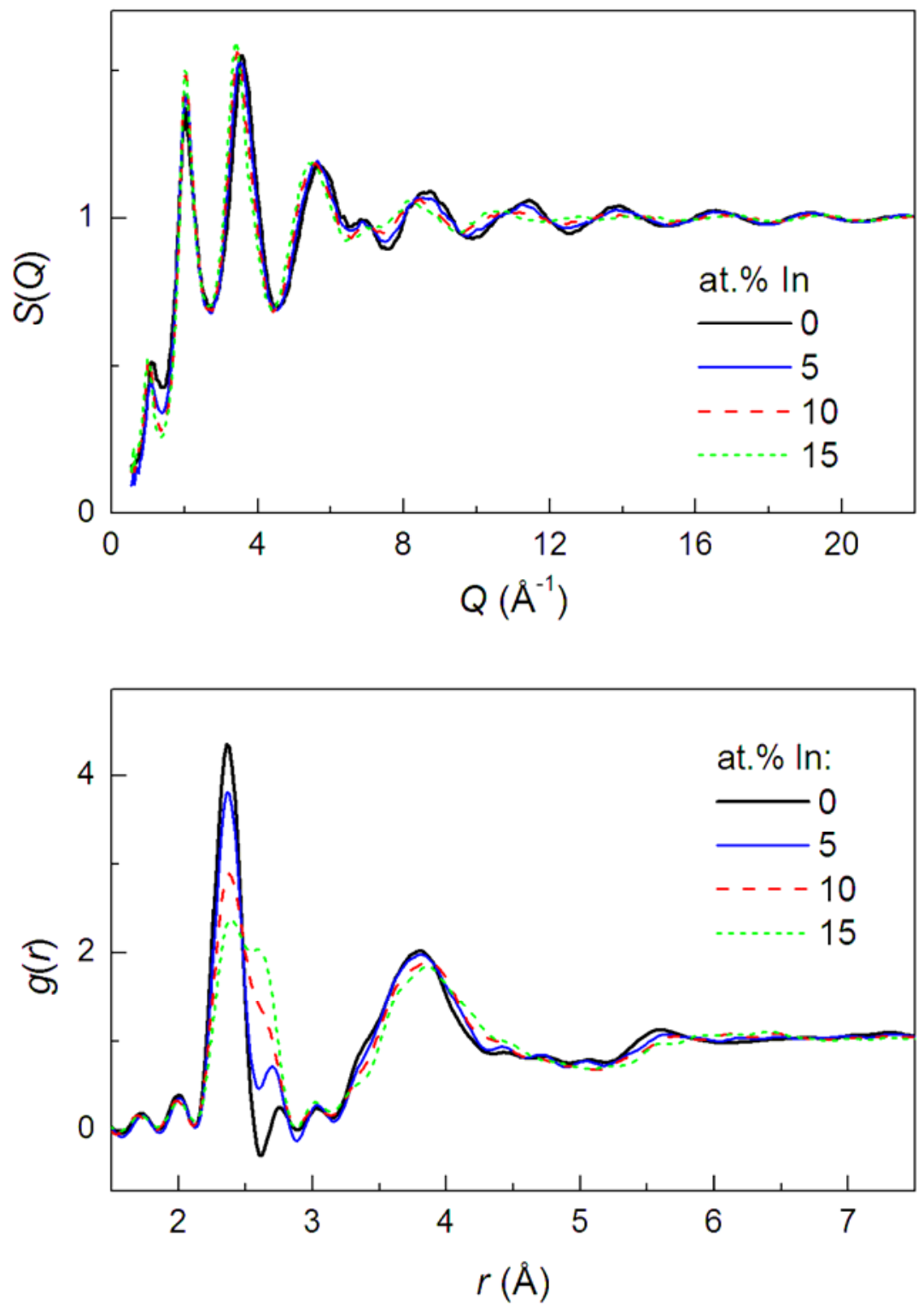

Figure 1. XRD total structure factors $S(Q)$ and pair distribution functions $g(r)$ for $\left(\mathrm{Ge}_{0.2} \mathrm{Se}_{0.8}\right)_{100-\mathrm{x}} \mathrm{In}_{\mathrm{x}}$ glasses. 

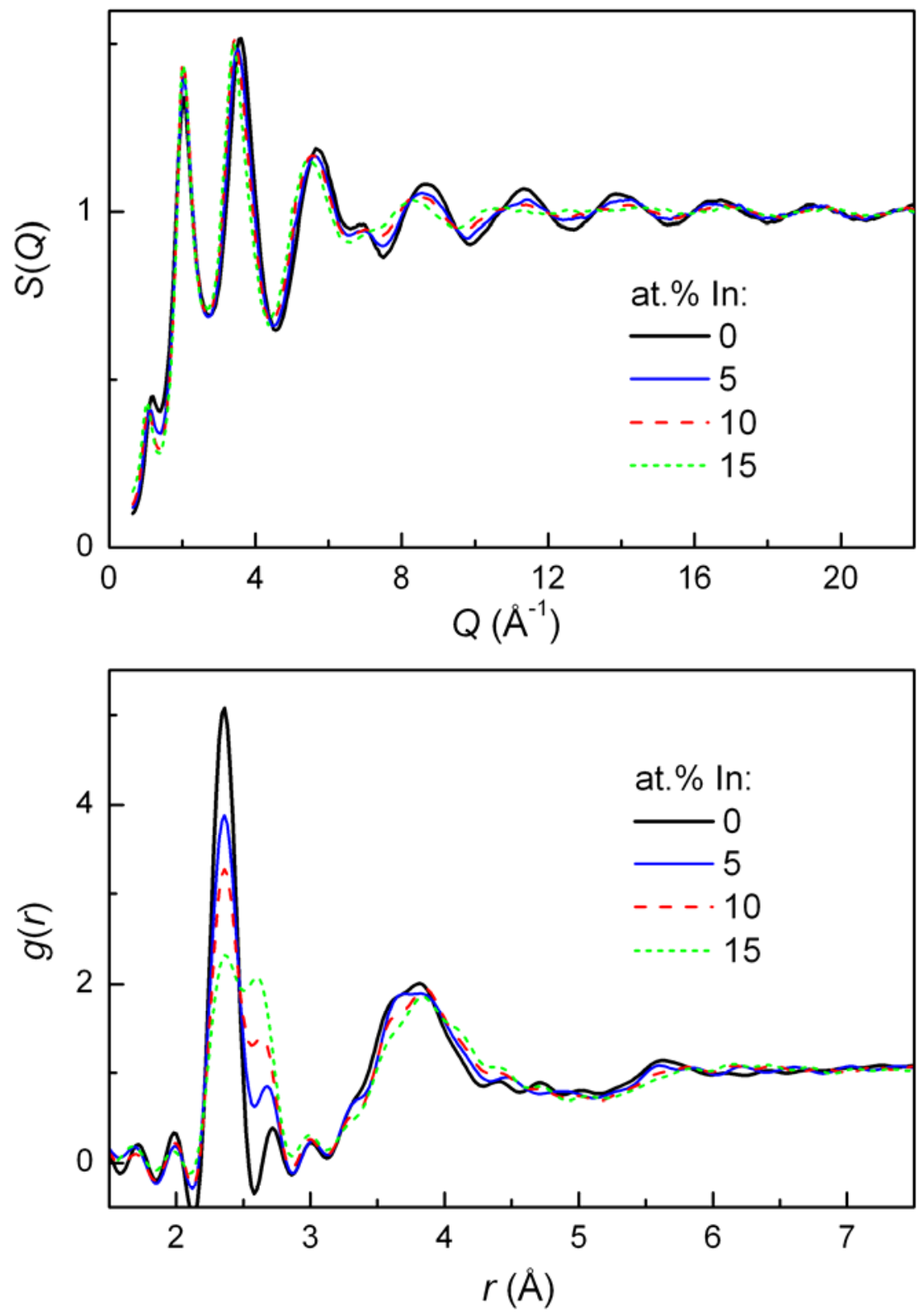

Figure 2. XRD total structure factors $S(Q)$ and pair distribution functions $g(r)$ for $\left(\mathrm{Ge}_{0.17} \mathrm{Se}_{0.83}\right)_{100-\mathrm{x}} \mathrm{In}_{\mathrm{x}}$ glasses. 

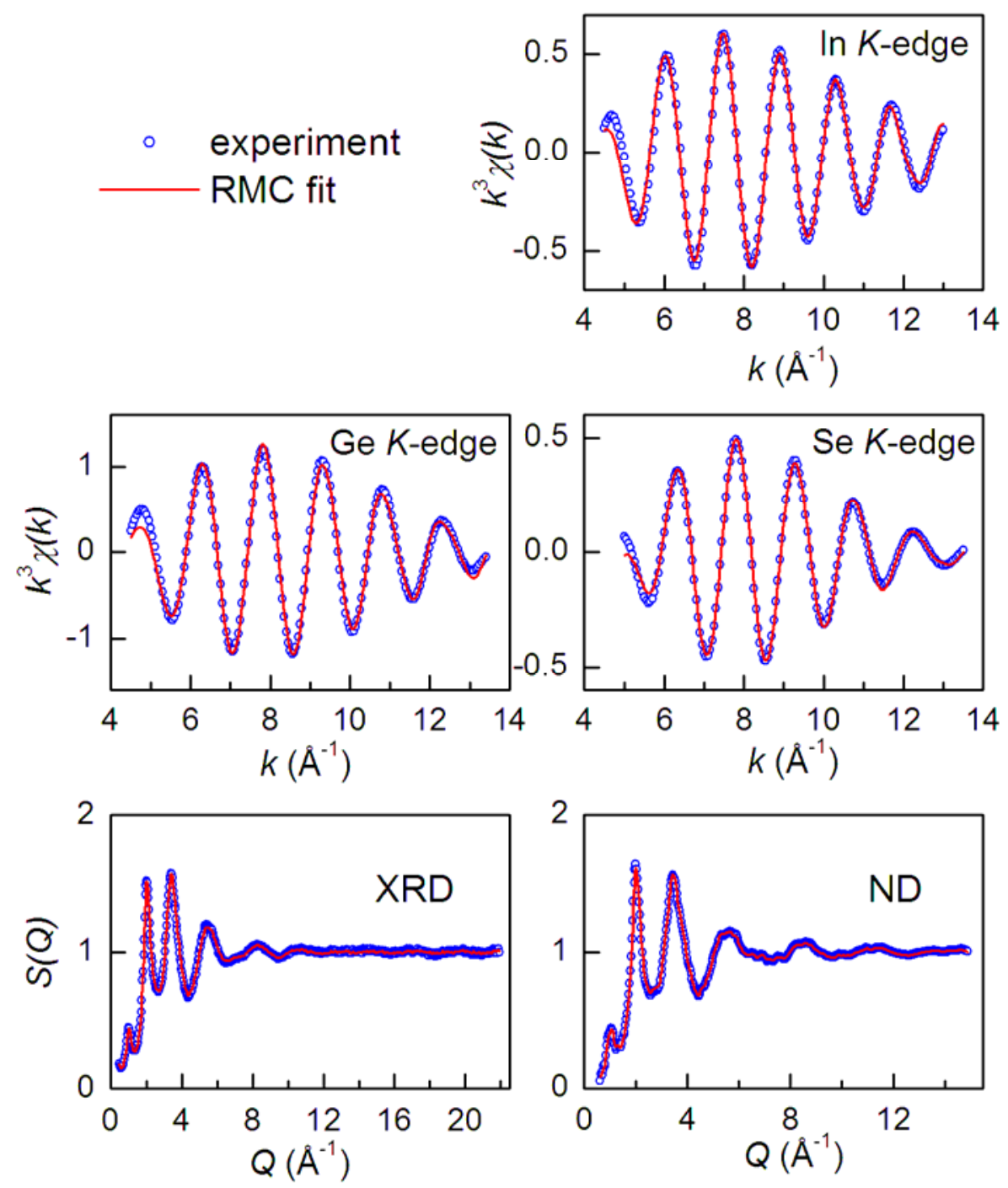

Figure 3. XRD and ND structure factors, and EXAFS spectra for $\left(\mathrm{Ge}_{0.17} \mathrm{Se}_{0.83}\right)_{85} \mathrm{In}_{15}$ glass: circles measurement; lines - data obtained by simultaneous RMC simulation of the experimental XRD, ND and EXAFS data. 

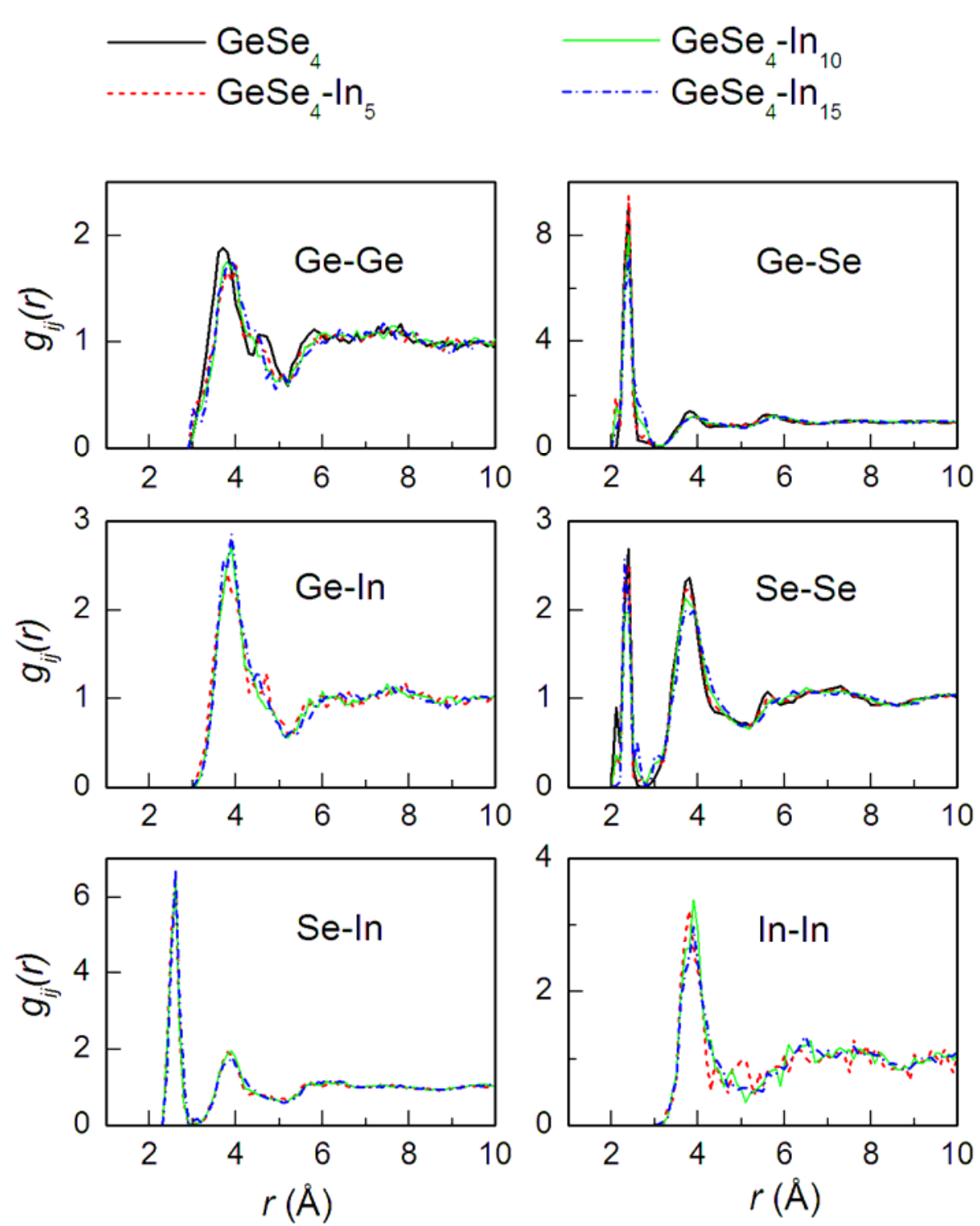

Figure 4. Partial pair distribution functions for $\left(\mathrm{Ge}_{0.2} \mathrm{Se}_{0.8}\right)_{100-\mathrm{x}} \mathrm{In}_{\mathrm{x}}$ glasses obtained with RMC. 

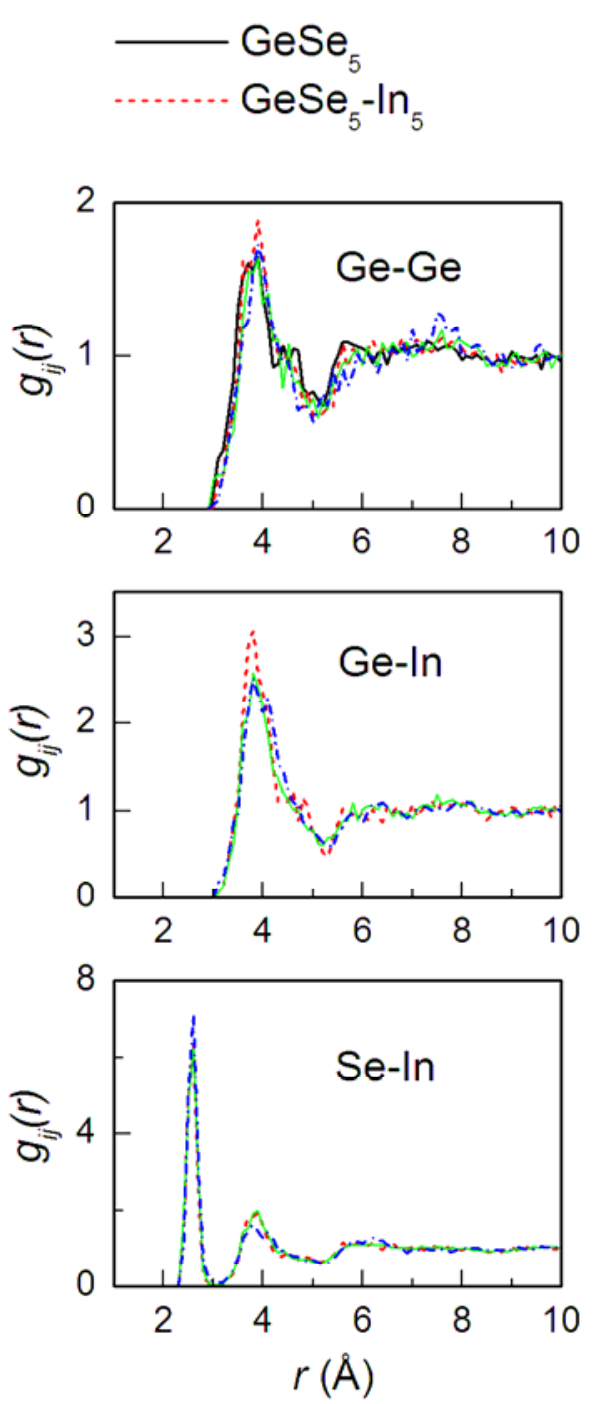
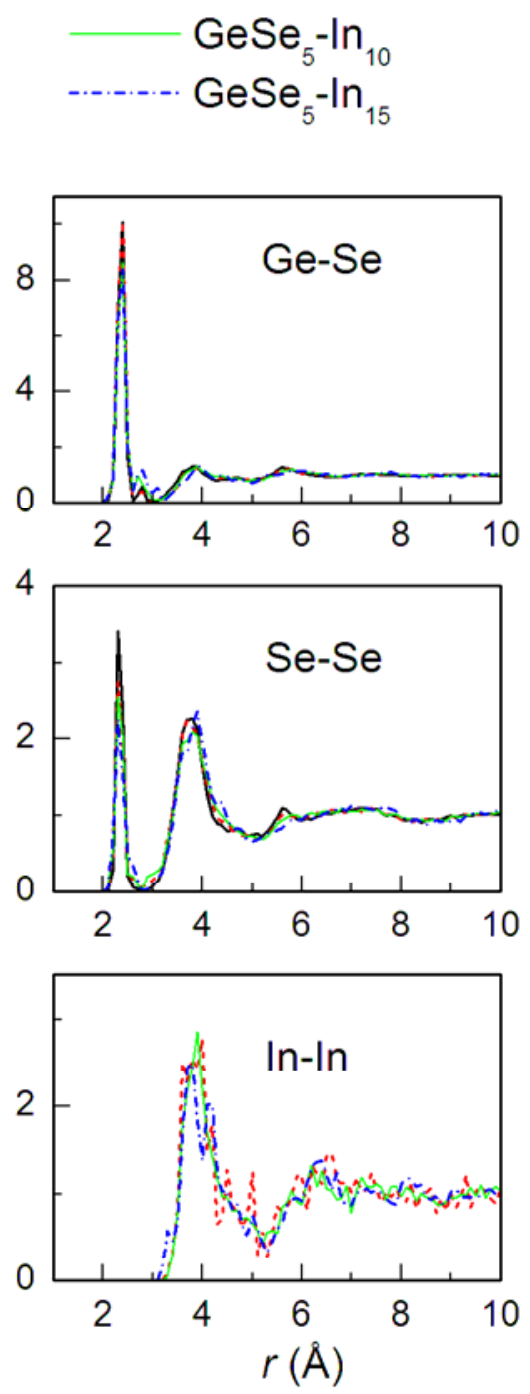

Figure 5. Partial pair distribution functions for $\left(\mathrm{Ge}_{0.17} \mathrm{Se}_{0.83}\right)_{100-\mathrm{x}} \mathrm{I} \mathrm{n}_{\mathrm{x}}$ glasses obtained with RMC.
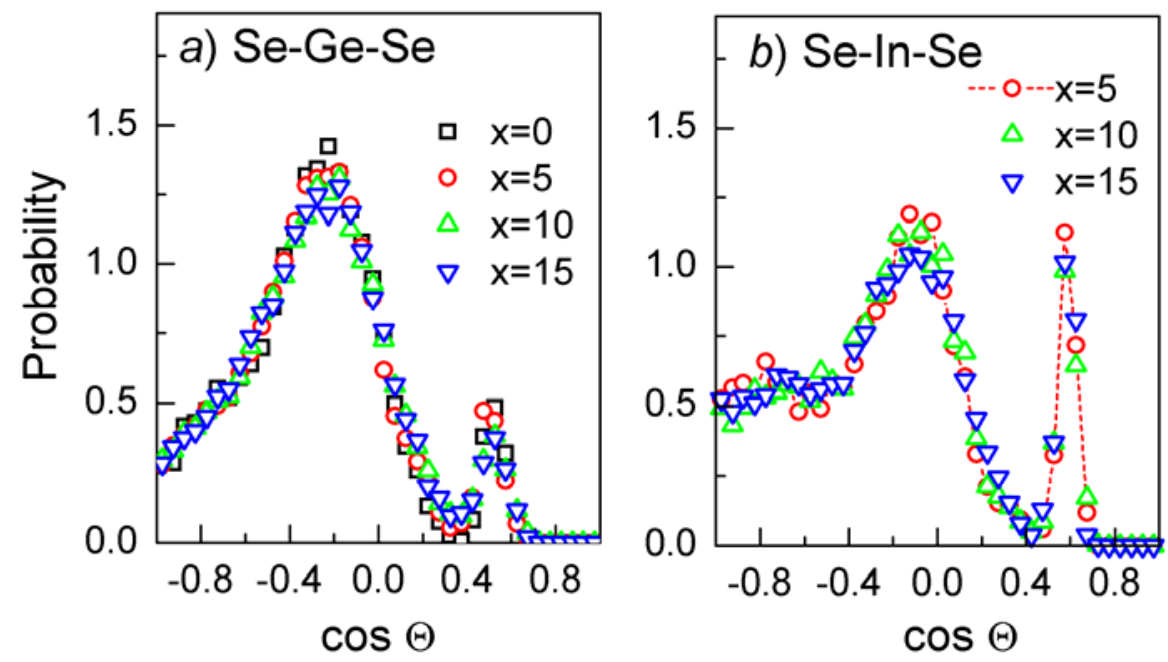

Figure 6. Se-Ge-Se (a) and Se-In-Se (b) bond angle distributions in $\left(\mathrm{Ge}_{0.2} \mathrm{Se}_{0.8}\right)_{100-\mathrm{x}} \mathrm{In}_{\mathrm{x}}$ glasses extracted from the RMC final atomic configurations. 